\title{
An Ocular Physiological Explanation for Visual Sensations in Near-Death Experiences
}

\author{
Einar Stefánsson, M.D.Ph.D. \\ Sindri Traustason, B.Sc. \\ Thor Eysteinsson, Ph.D. \\ University of Iceland and National University \\ Hospital, Reykjavik, Iceland
}

\begin{abstract}
The visual sensations in near-death experiences have been associated with disturbances in brain function, as well as religious and supernatural forces. We propose that these phenomena may be explained by the different tissue pressures in the eye and brain, which influence the consequences of reduced arterial blood pressure. The intraocular pressure is higher than the intracranial pressure, and therefore the perfusion pressure of the eye is lower than that of the brain proper. The eye will become ischemic and hypoxic earlier than the brain when arterial blood pressure falls, such as in death. The function of the retina may therefore be disturbed while the individual is still conscious, and this may result in visual sensations.
\end{abstract}

KEY WORDS: near-death experience; visual symptoms; intraocular pressure; eye; ocular physiology.

"Near-death experience" (NDE) is a collective term used for various sensations described by individuals who have died and been resuscitated. These experiences can be complex in nature and include visual, auditory, and emotional sensations, with images of structures, landscapes, and religious or secular figures (Osis and Haraldsson, 1977).

Einar Stefánsson, M.D., Ph.D., is Professor of Ophthalmology at the University of Iceland and the National University Hospital. Sindri Traustason, B.Sc., is a research student working on his master's thesis, and Thor Eysteinsson, Ph.D., is Associate Professor of Physiology, both at the University of Iceland. Reprint requests should be addressed to Dr. Stefánsson at the University of Iceland, Landspitali-háskólasjúkrahús, Reykjavik, Iceland; e-mail: einarste@landspitali.is. 
Reports of such experiences have often been interpreted as representations of the person's religious ideas, but studies have shown that incidence of NDEs is independent of the individual's religion (Osis and Haraldsson, 1977; van Lommel, van Wees, Meyers, and Elfferich, 2001).

Visual sensations are very prominent features of NDEs. Many experiencers describe visual hallucinations with the sensation of tunnels and lights. In general, they describe the sensation of looking into a tunnel with a bright light at the end, and sometimes visual hallucinations where they see images that they may have seen in everyday life. Scientists who have tried to explain these phenomena have tended to look at possible disturbances in the brain itself to explain these visual sensations and hallucinations, and have largely ignored the role of the eye in generating such images. The purpose of this article is to propose that these visual sensations may result from physiological changes in the eye, and specifically at the fact that the tissue pressure of the eye is higher than that of the brain and therefore the eye would experience ischemia sooner than the brain when arterial blood pressure falls.

Visual sensations similar to those reported in NDEs have been described by individuals suffering loss of consciousness as a result of cranial hypoxia or arterial hypotension caused by various factors. Pilots of high performance aircraft sometimes experience visual and auditory sensations similar to those in NDEs when excessive acceleration results in partial or complete loss of consciousness (Whinnery, 1997). Loss of peripheral vision is common in these situations (Shender, Forster, Hrebien, Ryoo, and Cammarota, 2003). Patients suffering from reflex anoxic seizures sometimes describe sensations similar to NDEs after waking up from seizures, including visions of tunnels and bright lights (Blackmore, 1998). Reflex anoxic seizures are caused by excessive activation of the vagus nerve resulting in a brief cardiac arrest. Experiments in this field show results that are consistent with studies of NDEs. T. Lempert, M. Bauer, and D. Schmidt (1994) induced syncope in 42 healthy young adults by using hyperventilation and the Valsalva maneuver, forced exhalation against the closed glottis, thus increasing intrathoracic pressure; 8 percent of their subjects described a vision of a tunnel.

\section{Ocular Physiology}

The tissue pressure of the eye, the intraocular pressure, is about 2 kilopascals $(\mathrm{kPa})$, or $15-16$ millimeters of mercury $(\mathrm{mmHg})$, which is 
higher than most tissues, including the brain, which has tissue pressure about $0.5-1.5 \mathrm{kPa}$, or $4-12 \mathrm{mmHg}$, depending on position (Pocock and Richards, 2004). The perfusion pressure of any organ is the mean arterial blood pressure minus the tissue pressure. The perfusion pressure of the eye is lower than the brain. This has little consequence when the arterial blood pressure is normal, but makes a difference in severe arterial hypotension, such as just before death. The perfusion pressure will reach zero in the eye before it does in the brain. The retina will become hypoxic and suffer disturbed function before the brain, when the arterial blood pressure declines before death. Additionally, the photoreceptors have perhaps the highest rate of oxygen consumption of any tissue in the body, and this will aggravate the hypoxic effect of ischemia ( $\mathrm{Yu}$, Cringle, and $\mathrm{Su}, 2005$ )

The retina receives blood from two vascular beds, the retinal circulation and the choroid. The inner part of the retina is supplied by the retinal circulation, whereas the choroid provides blood for the outer retina, including the photoreceptors. The retinal circulation is controlled by an autoregulatory mechanism, which dilates the vessels in hypoxia or arterial hypotension, whereas the choroid has no such mechanism. The peripheral retina is relatively thin, with only a single layer of retinal circulation, while the central part is thicker and has a more extensive circulation. The role of the choriocapillaris is proportionally greater in the peripheral part of the retina than in the center.

With arterial hypotension, the blood flow in the choroid is reduced in linear proportion to the perfusion pressure, and the photoreceptors in the outer retina would first suffer from hypoxia due to the reduced choroidal blood flow. The retinal circulation would supply the inner retina a little longer, due to the autoregulation. The brain would receive blood flow longer still, due to the lower tissue pressure and higher perfusion pressure. Therefore, the patient would suffer visual disturbances while still conscious. The tunnel effect might be due to the peripheral retina being a little more sensitive to the choroidal hypoxia than the central retina.

\section{Charles Bonnet Syndrome}

Once the eye has become fully ischemic and the retinal function ceases, the brain may still have some perfusion, as its tissue pressure is considerably lower than that of the eye. The situation is like that of a blind person with the brain still functioning. Charles Bonnet syndrome is named after naturalist, philosopher, and biologist Charles 
Bonnet (1720-1793) who in 1760 described hallucinations his blind grandfather experienced. The phenomenon is sometimes experienced by blind individuals or those with large blind spots in their visual fields. Those individuals in many cases experience structured hallucinations in the blind part of their visual field. They may see faces, gardens, landscapes, and other images that presumably come from their visual memory, as well as less structured images (Menkhaus, Wallesch, and Behrens-Baumann, 2003; Menon, Rahman, Menon, and Dutton, 2003). The source of these images must be the brain, even though the exact mechanism is unknown.

We propose that the visual experience in the NDE can be understood in light of the vascular physiology of the eye and the brain, and the well documented Charles Bonnet syndrome. Supernatural forces need not be implicated.

\section{References}

Blackmore, S. (1998). Experiences of anoxia: Do reflex anoxic seizures resemble neardeath experiences? Journal of Near-Death Studies, 17, 111-120.

Lempert, T., Bauer, M., and Schmidt, D. (1994). Syncope and near-death experience. Lancet, 344, 829-30.

Menkhaus, S., Wallesch, C. W., and Behrens-Baumann, W. (2003). [Charles-Bonnetsyndrome]. Ophthalmologe, 100, 736-9.

Menon, G. J., Rahman, I., Menon, S. J., and Dutton, G. N. (2003). Complex visual hallucinations in the visually impaired: the Charles Bonnet Syndrome. Survey of Ophthalmology, 48, 58-72.

Osis, K., and Haraldsson, E. (1977). At the hour of death. New York, NY: Avon.

Pocock, G., and Richards, C. (2004). Human physiology: The basis of medicine (2nd ed). New York, NY: Oxford University Press, pp. 132 and 340.

Shender, B. S., Forster, E. M., Hrebien, L., Ryoo, H. C., and Cammarota, J. P. (2003). Acceleration-induced near-loss of consciousness: the "A-LOC" syndrome. Aviation, Space, and Environmental Medicine, 74, 1021-8.

van Lommel, P., van Wees, R., Meyers, V., and Elfferich, I. (2001). Near-death experience in survivors of cardiac arrest: A prospective study in the Netherlands. Lancet, 358, 2039-45.

Whinnery, J. E. (1997). Psychophysiologic correlates of unconsciousness and near-death experiences. Journal of Near-Death Studies, 15, 231-258.

Yu, D. Y., Cringle, S. J., and Su, E. N. (2005). Intraretinal oxygen distribution in the monkey retina and the response to systemic hyperoxia. Investigative Ophthalmology and Visual Science, 46, 4728-33. 